\title{
Avaliação Comparativa das Perdas de Água por Evapotranspiração em Mesocosmos Colonizados POR Diferentes MACRÓFITAS AQUÁTICAS ${ }^{1}$
}

\author{
Comparative Evaluation of Water Losses by Evapotranspiration in Mesocosms Colonized by \\ Different Aquatic Weeds
}

ROSA, C.S. ${ }^{2}$, ANTUNES, R.D. ${ }^{3}$, PITELLI, R.A. ${ }^{4}$ e PITELLI, R.L.C.M. ${ }^{5}$

\begin{abstract}
RESUMO - Uma das mais importantes formas de interferência das macrófitas aquáticas em corpos hídricos é o aumento das perdas d'água para a atmosfera pela evapotranspiração. Em alguns corpos hídricos esse aumento pode ser considerado uma interferência negativa e indesejável, especialmente em reservatórios para armazenamento de água e reservatórios de acúmulo para geração de energia elétrica. Assim, este trabalho foi realizado com o objetivo de comparar as perdas d'água em mesocosmos colonizados com macrófitas aquáticas importantes em corpos hídricos no Brasil, mais especificamente Myriophyllum aquaticum, Brachiaria subquadripara, Echinochloa polystachya, Typha latifolia e Pontederia lanceolata. As avaliações foram conduzidas no mês de julho de 2004, em três periodos de quatro dias. As perdas d'água foram avaliadas pelas quantidades necessárias para reposição do nível existente antes do respectivo período de avaliação. A colonização de macrófitas aquáticas aumentou as perdas d'água nos mesocosmos, com maiores valores observados naqueles colonizados por T. latifolia: entre 3,54 e 4,71 vezes a superfície de água sem macrófitas. As perdas nos mesocosmos colonizados por M. aquaticum, B. subquadripara, E. polystachya e P. lanceolata foram estatisticamente similares e promoveram aumentos entre $1,54 \mathrm{e}$ 2,21 vezes a superficie livre. Os resultados evidenciam a importância do manejo de macrófitas aquáticas em corpos hídricos para armazenamento de água.
\end{abstract}

Palavras-chave: Myriophyllum aquaticum, Brachiaria subquadripara, Echinochloa polystachya, Typha latifolia, Pontederia lanceolata.

\begin{abstract}
Water loss increase through evapotranspiration is one the most important modes of interference of weeds in water bodies, especially in water storage reservoirs. Thus, this research was conducted to compare the water losses in mesocosms colonized with the aquatic weeds Myriophyllum aquaticum, Brachiaria subquadripara, Echinochloa polystachya, Typha latifolia and Pontederia lanceolata. The evaluations were carried out in July 2004, in three 4-day periods. The water losses were evaluated based on the amounts of waternecessary to restore the existing water level before each evaluation period. The colonization of the aquatic weeds increased the water losses in the mesocosms, with the highest losses being observed in those colonized by T. latifolia, between 3.54 to 4.71 times the water surface without aquatic weeds. The losses in the mesocosms colonized by M. aquaticum, B. subquadripara, $\boldsymbol{E}$. polystachya, and $\boldsymbol{P}$. lanceolata were statistically similar and promoted increases between 1.54 to 2.21 times the free surface. The results showed that aquatic weed control is important to prevent water losses in reservoirs used for water storage.
\end{abstract}

Keywords: Myriophyllum aquaticum, Brachiaria subquadripara, Echinochloa polystachya, Typha latifolia, Pontederia lanceolata.

1 Recebido para publicação em 10.7.2008 e na forma revisada em 21.8.2009.

2 Engōo-Agroo , Bromélias Rio, <camisrosa@yahoo.com.br>; ${ }^{3}$ Engo-Agr ${ }^{\circ}$., Syngenta Proteção de Cultivos Ltda., $<$ ricardo.antunes@syngenta.com>; ${ }^{4}$ Professor Titular, Dep. de Fitossanidade, UNESP, Jaboticabal-SP, <rapitelli@ecosafe.agr.br>;

${ }^{5}$ Engo-Agr ${ }^{\circ}$., Dr., Ecosafe Agricultura e Meio Ambiente S/C Ltda., Jaboticabal-SP, <rlpitelli@ecosafe.agr.br>. 


\section{INTRODUÇÃO}

O aumento das perdas de água pela evapotranspiração é uma das mais importantes interferências negativas ocasionadas por extensas e densas colonizações de corpos hídricos por macrófitas emersas e emergentes (Pitelli, 1998). No início do século 20, Otis (1914) observou que áreas colonizadas com macrófitas aquáticas normalmente apresentavam maiores perdas de água quando comparadas com a superficie livre de água. Esse autor observou grande diferença entre áreas colonizadas com diversas macrófitas e a atribuiu às diferenças da morfologia interna e externa das espécies. Brezny et al. (1973) discutiram vários trabalhos, evidenciando a importância dos fatores climáticos na relação das perdas d'água entre áreas colonizadas com macrófitas e áreas com superficie livre. Esses autores citam publicação de Seybold (1930) citado por (Brezny et al., 1973), a qual mostra que, à medida que a velocidade de vento aumenta, as perdas de água aumentam mais intensamente na superficie livre do que em áreas colonizadas por Lemna minor. Brezny et al. (1973), por outro lado, observaram correlações positivas entre velocidade do vento, temperaturas máximas e evapotranspiração em áreas colonizadas por C. rotundus, Typha angustifolia, Ipomoea aquatica e Eichhornia crassipes. Em áreas colonizadas com Trapa natans, os autores apenas observaram correlação entre temperatura máxima e evapotranspiração. Considerando que são plantas com diferentes hábitos de crescimento, algumas com folhas com disposição ereta em posição oblíqua à superfície d'água, como as dos gêneros Typha, Pontederia e Eichhornia, outras com folhas dispostas sobre a superficie, como as dos gêneros Lemna trappa, e outras com folhas dispostas em roseta e bastante pilosas, com $P$. stratiotes, os resultados evidenciam a importância da forma da planta e da estrutura de suas colonizações em relação às perdas d'água por evapotranspiração. Todas elas são plantas bastante conhecidas como macrófitas aquáticas importantes em corpos hídricos no Brasil e em regiões tropicais e subtropicais do mundo, algumas consideradas entre as piores plantas daninhas aquáticas do Brasil, como ressalta Pitelli (1998).
Boyd (1987), estudando tanques colonizados por Justicia americana, Alternanthera philoxeroides e Juncus effusus, não constatou relação entre os índices de área foliar desenvolvidos por cada uma das três espécies e as perdas d'água por evapotranspiração. Esse autor considera que as características morfofisiológicas da folha influenciaram em alto grau a taxa de transpiração, mascarando os efeitos da magnitude da área foliar.

Uma das abordagens comparativas mais comuns em estudos de demandas evapotranspiratórias de comunidades vegetais é a relação entre as perdas d'água por evapotranspiração de superficies colonizadas por determinadas plantas $\left(E P_{i}\right)$ e a evaporação da superficie correspondente sem plantas $\left(\mathrm{TR}_{\mathrm{i}}\right)$, ou seja, $\mathrm{EVP}_{\mathrm{i}} / \mathrm{TR}_{\mathrm{i}}$. Os valores observados na literatura consultada variam bastante em função de vários fatores climáticos e das condições das plantas avaliadas. As faixas de valores para algumas espécies foram: Azolla caroliniana: 0,40 - 2,50 (Otis, 1914; Bernatowicz et al., 1976; Lallana et al., 1987); A. philoxeroides: 1,26 (Boyd, 1987); Carex luria: 1,63 (Boyd, 1987); C. rotundus: 2,31 - 2,50 (Brezny et al., 1973); E. crassipes: 1,02-4,50 (Otis, 1914; Timmer \& Weldon, 1967; Brezny et al.,1973; van der Weert \& Kamerling, 1974; Lallana et al., 1987; Snyder \& Boyd, 1986; Castro, 2008); I. aquatica: 1,01 - 1,24 (Brezny et al., 1973); Juncus effusus: 1,52 (Boyd, 1987); J. americana: 1,17 (Boyd, 1987); Nymphaea odorata: 1,00 (Otis, 1914); Panicum rigidulum: 1,58 (Boyd, 1987); Pistia stratiotes: 0,79 - 1,75 (Brezny et al., 1973; Lallana et al., 1987); Pontederia cordata: 1,20 (Otis, 1914); Potamogeton nodosus: 3,10 (Otis, 1914); S. herzogii: 0,90 - 1,60 (Lallana et al., 1987); Scirpus validus: 1,90 (Otis, 1914); T. natans: 0,93 - 1,03 (Brezny et al., 1973); Typha angustifolia: 1,27 - 1,67 (Brezny et al., 1973); e T. latifolia: 1,75 - 2,00 (Otis, 1914; Snyder \& Boyd, 1986).

Com o objetivo de avaliar as demandas evapotranspiratórias de superficies de corpos hídricos colonizados por Myriophyllum aquaticum, Brachiaria subquadripara, Echinochloa polystachya, T. dominguensis e Pontederia lanceolata, foi conduzido o presente trabalho, em condições de mesocosmos, durante o período seco na região de Jaboticabal, SP. A grande justificativa para realização deste 
trabalho é a retomada desse tipo de estudo, bastante realizado no passado em outras condições, e atualizá-lo com plantas de importância mais recente no Brasil, como B. subquadripara e $M$. aquaticum. É importante destacar que se trata de um trabalho de pequeno porte que visa fazer com que outros pesquisadores se interessem pelo tema e realizem pesquisas locais, uma vez que, tal qual a evaporação, as demandas evapotranspiratórias dependem de condições locais. Mais importante ainda é ponderar que mais estudos desse tipo devem ser realizados, visando fornecer subsídios para estimar as perdas de água por evapotranspiração em corpos hídricos, especialmente aqueles destinados ao armazenamento de água.

\section{MATERIAL E MÉTODOS}

O ensaio foi conduzido no Núcleo de Estudos e Pesquisas Ambientais em Matologia (Nepeam), Unesp, Jaboticabal, SP. As macrófitas foram cultivadas em mesocosmos com capacidade de $0,81 \mathrm{~m}^{3}$, superficie de $2,55 \mathrm{~m}^{2} \mathbf{e}$ sedimento formado por uma camada de $0,15 \mathrm{~m}$ de mistura composta por terra, areia e turfa $(2: 1: 1)$. A lâmina de água foi de $30 \mathrm{~cm}$. O delineamento experimental adotado foi inteiramente casualizado, com quatro repetições. Cada mesocosmo foi considerado uma unidade experimental. Mesocosmos sem colonização com macrófitas aquáticas foram mantidos como testemunhas. As plantas aquáticas foram mantidas nos mesocosmos até que colonizassem acima de $70 \%$ da superficie da lâmina d'água, quando foram iniciadas as avaliações.

Para o presente estudo, foram considerados os mesocosmos colonizados com Myriophyllum aquaticum, Brachiaria subquadripara, Echinochloa polystachya, Typha latifolia e $P$. lanceolata, sendo as únicas espécies que atingiram a taxa de colonização estabelecida ( $75 \%$ da superfície da lâmina d'água).

As avaliações de perdas de água foram realizadas em três períodos de quatro dias, em junho de 2004. O experimento foi conduzido por um período de dois meses, mas foram descartados os períodos com chuvas ou orvalho matinal. No começo de cada período, o nível de água foi estabelecido em cada mesocosmo. No final do período correspondente, a água foi reposta em volumes conhecidos, até que o nivel original fosse restabelecido. A quantidade de água reposta foi considerada como as perdas evapotranspiratórias do sistema no período.

Considerando o possivel "efeito-oásis" que ocorreu nos mesocosmos, a pertinência dos resultados obtidos reside nas comparações entre os mesocosmos colonizados com diferentes espécies e não nos valores absolutos obtidos. Assim, todos os dados foram expressos pelas relações entre as perdas de água nos mesocosmos com macrófitas (EVTi) e as perdas na testemunha sem macrófitas (EVi). A análise de variância dos dados foi realizada pelo teste $\mathrm{F}$, e as comparações das médias, pelo teste de Tukey.

\section{RESULTADOS E DISCUSSÃO}

Na Figura 1 estão graficamente apresentados os valores obtidos nos cálculos dos valores da relação $\mathrm{EVP}_{\mathrm{i}} / \mathrm{EV}_{\mathrm{i}}$ observados para mesocosmos colonizados com as diferentes macrófitas aquáticas, nas três épocas de avaliação. A constatação mais importante desta figura é de que em todos os mesocosmos colonizados pelas macrófitas ocorreram perdas d'água superiores às do mesocosmo testemunha, com valores da relação $\mathrm{EVP}_{\mathrm{i}} / \mathrm{EV}_{\mathrm{i}}$ maiores que a unidade.

As perdas d'água do sistema colonizado com $T$. latifolia foram significativamente maiores que as observadas nos mesocosmos colonizados com as outras macrófitas, com os valores da relação $\mathrm{EVP}_{\mathrm{i}} / \mathrm{EV}_{\mathrm{i}}$ variando entre 3,54 e 4,71. Esses valores foram maiores que os observados por Otis (1914) e Snyder \& Boyd (1986) com essa mesma macrófita aquática e os observados em mesocosmos colonizados com T. angustifolia (Brezny et al., 1973). A forma da colonização da taboa permite movimentação de ar e penetração de radiação solar, facilitando o processo de evapotranspiração do corpo hídrico.

Não foram verificadas diferenças estatisticamente significativas entre as perdas d'água dos mesocosmos colonizados com E. polystachya, M. aquaticum, P. lanceolata e 


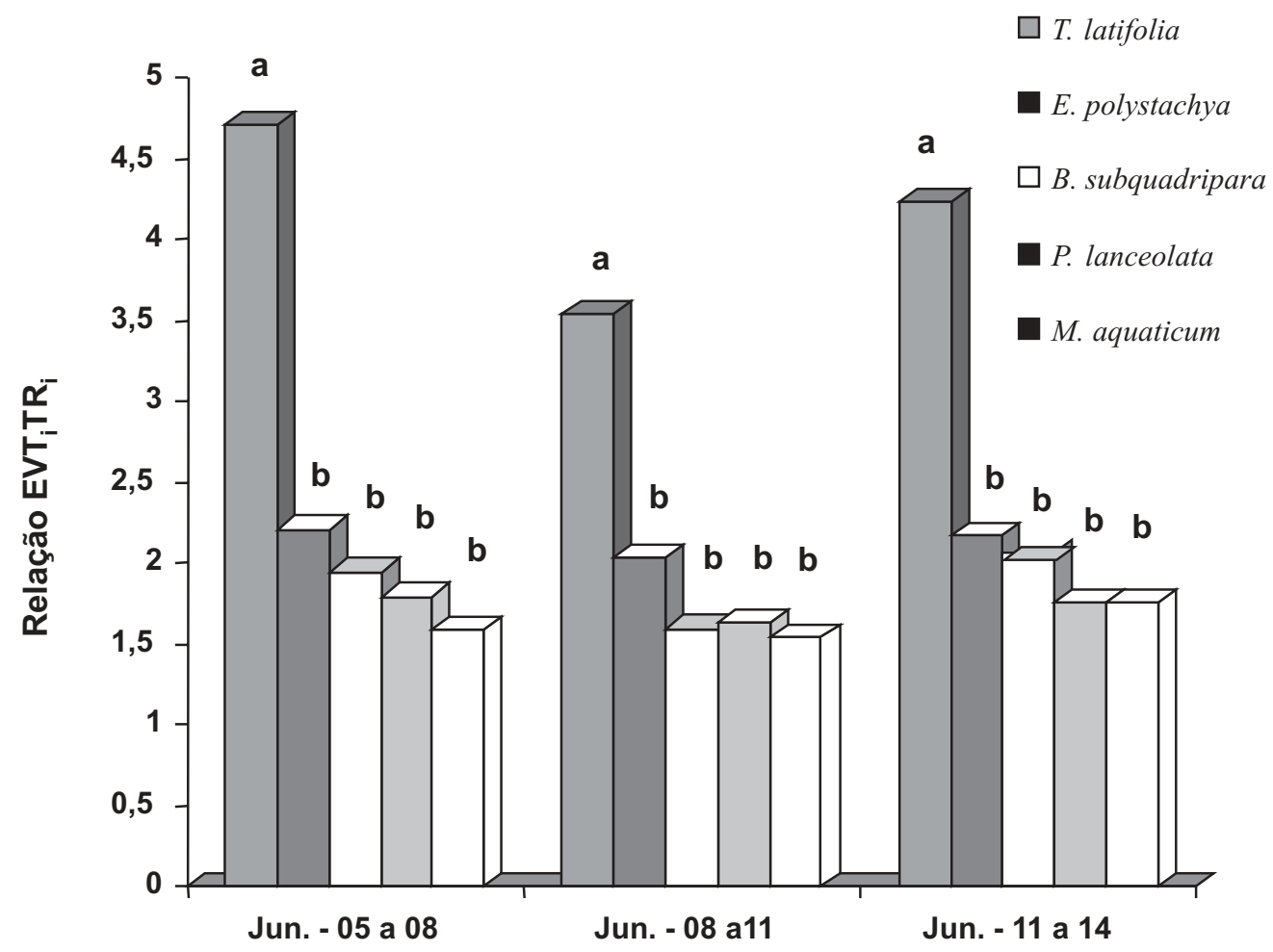

Figura 1 - Valores da relação entre as perdas d'água por evapotranspiração de mesocosmos colonizados por macrófitas aquáticas e por evaporação em mesocosmos sem a presença de plantas.

B. subquadripara. Os valores da relação $\mathrm{EVP}_{\mathrm{i}} /$ $\mathrm{EV}_{\text {i }}$ para essas espécies variaram entre 1,54 e 2,21, indicando que essas macrófitas aquáticas incrementaram as perdas d'água entre $54 \%$ e $121 \%$ em relação à superfície de água. Os resultados permitem inferir que colonizações de macrófitas aquáticas, quando acima da capacidade suporte do ambiente para suas populações, podem incrementar as perdas d'água, com consequências nas disponibilidades para corpos hídricos localizados a jusante.

Os valores observados estão dentro da faixa daqueles encontrados para $A$. caroliniana (Otis, 1914; Bernatowicz et al., 1976; Lallana et al., 1987), A. philoxeroides (Boyd, 1987), Carex luria (Boyd, 1987), E. crassipes (Otis, 1914; Timmer \& Weldon, 1967; Brezny et al., 1973; van der Weert \& Kamerling, 1974; Lallana et al., 1987; Snyder \& Boyd, 1986), Pistia stratiotes (Brezny et al., 1973; Lallana et al., 1987), S. herzogii (Lallana et al., 1987) e S. validus (Otis, 1914). Eles foram superiores aos valores encontrados para Ipomoea aquatica
(Brezny et al., 1973), J. effusus (Boyd, 1987), J. americana (Boyd, 1987), N. odorata (Otis, 1914), P. cordata (Otis, 1914) e T. natans (Brezny et al., 1973).

Para melhor entendimento dessa forma de interferência das macrófitas aquáticas em corpos hídricos e estabelecer uma magnitude do processo, foi realizada uma simulação utilizando dados publicados em Brasil (2008), no qual consta que a média das perdas d'água por evaporação em corpos hídricos do semiárido da Bahia é ao redor de $2.000 \mathrm{~L} \mathrm{~m}^{-2}$ por ano. Para simulação, será considerado o reservatório de Pedras Altas, localizado na região de Senhor do Bonfim, BA, o qual tem uma superfície média de 750 ha de lâmina d'água e, portanto, uma perda de $15.000 .000 \mathrm{~m}^{3}$ por ano apenas por evaporação. Esse reservatório tem uma população de E. crassipes cuja área de colonização é variável com a estação, apresentando uma média anual de $20 \%$ da superficie da lâmina d'água. Considerando um valor médio da faixa de valores observados por Lallana et al. (1987) na Argentina como o aumento 
médio das perdas por evapotranspiração, ou seja, 2,78 vezes a superficie livre, a perda de água do reservatório de Pedras Altas passará para $20.340 .000 \mathrm{~m}^{3}$ por ano, isto é, $5.340 .000 \mathrm{~m}^{3}$ adicionais. Se nas áreas mais rasas o reservatório tiver uma colonização de $5 \%$ com T. latifolia, e considerando uma relação EVPi/ EVi igual a 4,13 para áreas colonizadas com esta macrófita, haverá uma perda total de água de $22.687 .750 \mathrm{~m}^{3}$ por ano no reservatório. Considerando apenas essas duas macrófitas, as perdas d'água do reservatório de Pedras Altas, passarão a ter um valor $51,25 \%$ maior que se houvesse plantas aquáticas em tais condições de colonização.

As perdas adicionais nesse reservatório pela evapotranspiração são bastante expressivas, considerando que não haja excesso de água que extravase a capacidade de armazenamento desse açude e que esse corpo hídrico seja utilizado para armazenamento de água para dessedentação da população e de animais.

Conclui-se que a colonização por qualquer das macrófitas aquáticas estudadas aumentou as perdas d'água dos mesocosmos, sendo os maiores valores observados para T. latifolia, quando comparada com E. polystachya, $B$. subquadripara, $P$. lanceolata e $M$. aquaticum. Recomenda-se que, para qualquer análise do risco/benefício de controle de macrófitas aquáticas, as perdas d'água por evapotranspiração sejam consideradas, desde que não sejam reservatórios de fio-d'água (que não necessita de acúmulo de água para uso na estação seca) ou em época em que não haja restrição hídrica.

\section{LITERATURA CITADA}

BERNATOWICZ, S.; LESZCYNSKY, S.; TYCZNSKA, S.

The influence of transpiration by emergent plants on the water balance in lakes. Aquatic Bot., v. 2, n.2, p. 275-278, 1976.
BOYD, C. E. Evapotranspiration/evaporation (EVo/Eo) ratios for aquatic plants. J. Aquatic Weed Manag., v. 25. n. 1, p. 1-3, 1987.

BRASIL. Região de planejamento e gestão das águas. Sobradinho. Disponível em: <www.srh.ba.gov.br/appsrh/ publicacoes/gestão/Evaporacao.acudessemiarido $>$. Acesso em: 14 nov. 2008.

BREZNY, O.; MEHTA, I.; SHARMA, R. K. Studies on evapotranspiration of some aquatic weeds. Weed Sci., v. 21, p. 197-204, 1973.

CASTRO, R. M. Estudo comparativo das perdas d'água em mesocosmos colonizados ou não por aguapé (Eichhornia crassipes (Mart.) Solms-Laubach). 2008. 77 f. Dissertação (Mestrado em Agricultura) - Universidade Estadual Paulista, Botucatu, 2008.

LALLANA, V. H.; SABATTINI, R. A.; LALLANA, M. D. C. Evapotranspiration from Eichhornia crassipes, Pistia stratiotes, Salvinia herzogii and Azolla caroliniana during summer in Argentina. J. Aquat. Plant Manag., v. 25, n. 1, p. $48-50,1987$

OTIS, C. H. The transpiration of emerged water plants: its measurements and relationships. Bot. Gaz., v. 58, p. 457-494, 1914.

PITELLI, R. A. Macrófitas aquáticas no Brasil na condição de problemáticas. In: WORKSHOP SOBRE CONTROLE DE PLANTAS AQUÁTICAS, 1998, Brasília. Anais... Brasília: Ministério da Agricultura/IBAMA, 1998. p. 12-15.

SNYDER, R. L., BOYD, C. E. Evapotranspiration of Eichhornia crassipes (Mart) Solms and Typha latifolia L. Aquatic Bot., v. 27, p. 217-227, 1987.

TIMMER, C. E.; WELDON, L. W. Evapotranspiration and pollution of waterhyacinth. Hyacinth Control J., v. 6, n 1, p. $3437,1967$.

van der WEERT, R.; KAMERLING, G. E. Evapotranspiration of water hyacinth (Eichhornia crassipes). J. Hydrol., v. 22, p. 201-212, 1974. 